\title{
Crystal and Cryo-EM structures provide insight into how pro-neurodegenerative SARM1 is activated and cleave $\mathrm{NAD}^{+}$
}

\author{
T. Ve ${ }^{1}$, W. Gu ${ }^{2}$, J. Nanson ${ }^{2}$, Y.Shi ${ }^{1}$, K. Cunnea ${ }^{3}$, P. Kerry ${ }^{3}$, T. Bosanac ${ }^{4}$, R. Hughes ${ }^{4}$ and B. Kobe ${ }^{2}$ \\ ${ }^{1}$ Institute for Glycomics, Griffith University, Southport, QLD 4222, Australia. \\ ${ }^{2}$ School of Chemistry and Molecular Biosciences, University of Queensland, QLD 4072, Australia. \\ ${ }^{3}$ Evotec Ltd, 114 Innovation Drive, Milton Park, Abingdon, Oxfordshire, OX14 4RZ, UK. \\ ${ }^{4}$ Disarm Therapeutics, a wholly-owned subsidiary of Eli Lilly \& Co., Cambridge, MA, USA.
}

t.ve@griffith.edu.au

Axonal degeneration is responsible for disease progression and accumulation of disability in many neurodegenerative conditions. Sterile alpha and Toll/interleukin-1 receptor motif-containing 1 (SARM1) is a nicotinamide adenine dinucleotide (NAD ${ }^{+}$)- cleaving enzyme whose activation triggers axon destruction [1-4]. Loss of the biosynthetic enzyme NMNAT2, which converts nicotinamide mononucleotide $(\mathrm{NMN})$ to $\mathrm{NAD}^{+}$, activates SARM1 via an unknown mechanism. Using crystallography, cryo-EM, NMR and biochemical assays, we demonstrate that SARM1 is activated by an increase in the ratio of NMN to $\mathrm{NAD}^{+}$and show that both metabolites compete for binding to the autoinhibitory N-terminal armadillo repeat (ARM) domain of SARM1 [5]. We show that NMN binding disrupts ARM-TIR interactions in the full-length SARM1 octamer, enabling its TIR domains to self-associate and form a catalytic site capable of cleaving $\mathrm{NAD}^{+}$[5]. These structural insights identify SARM1 as a metabolic sensor of the NMN/NAD ${ }^{+}$ratio, define the mechanism of SARM1 activation, and may enable a path to the development of allosteric inhibitors that block SARM1 activation.

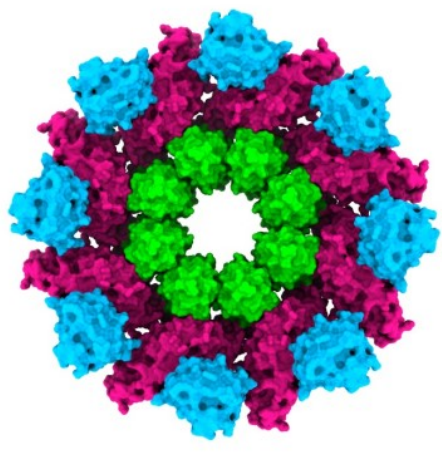

\section{$[\mathrm{NMN}] /\left[\mathrm{NAD}^{+}\right] \uparrow$}

$\mathrm{NAD}^{+}$displaced by NMN
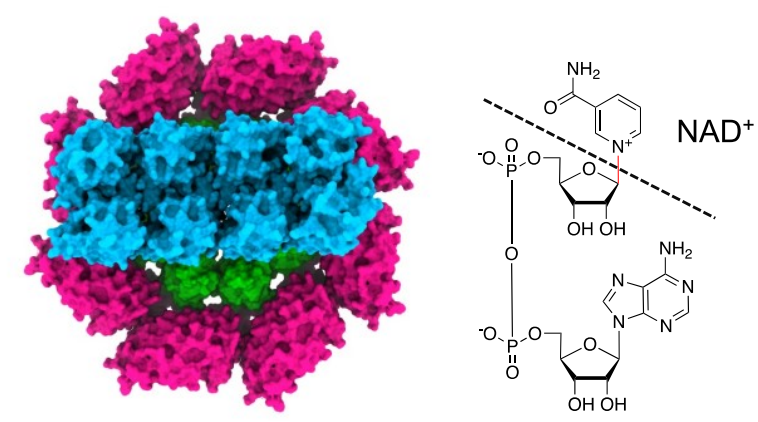

ARM

SAM

TIR

Figure 1. SARM1 activation mechanism. Left: Inactive cryo-EM structure of SARM1. Right: Model of activated SARM1 with oligomerised TIR domains capable of cleaving $\mathrm{NAD}^{+}$.

[1] Essuman, K. et al.(2017). Neuron 93, 1334-1343.

[2] Essuman, K. et al. (2018). Curr. Biol. 28, 421-430.

[3] Horsefield, S. et al. (2019). Science 365, 793-799.

[4] Wan, L. et al. (2019). Science 365, 799-803.

[5] Figley, M. et al. (2021). Neuron 109, 1118-1136.

Keywords: NADase; TIR domain; ARM domain; allostery; cryo-EM; X-ray crystallography 